\title{
PLIF for the treatment of adult spinal deformity
}

\author{
Michael Y. Wang
}

Received: 30 November 2010 / Accepted: 1 December 2010/Published online: 16 December 2010

(C) Springer-Verlag 2010

In this article, Tsai and colleagues review their results using posterior interbody fusion (PLIF) to treat 58 patients with degenerative scoliosis. The report is notable in that the authors had excellent follow-up and utilized accepted outcomes measures to ascertain the degree of clinical improvement in their cohort, and I congratulate them on their excellent outcomes. As neurosurgeons increasingly treat elderly patients with deformity, careful consideration of elements traditionally under the orthopedist's purview must be entertained. This includes the factors of spinal balance, complex decision making for long-construct planning, fixation into osteoporotic bone, and the prolonged postoperative management of these difficult patients.

The technique applied in this report is familiar to neurosurgeons: a modification of the original PLIF technique devised by the late American neurosurgeon Ralph Cloward. The PLIF technique actually represents a powerful method for decompressing, mobilizing, and stabilizing a single lumbar motion segment circumferentially. Thus, its

M. Y. Wang $(\bowtie)$

Department of Neurological Surgery,

University of Miami Miller School of Medicine,

Lois Pope LIFE Center, 2nd Floor, 1095 NW 14th Terrace (D4-6),

Miami, FL 33136, USA

e-mail: MWang2@med.miami.edu

M. Y. Wang

Department of Rehabilitation Medicine,

University of Miami Miller School of Medicine,

Lois Pope LIFE Center, 2nd Floor, 1095 NW 14th Terrace (D4-6),

Miami, FL 33136, USA appeal to neurosurgeons as a starting point for deformity management is obvious. The main drawbacks of multi-level PLIF are that (1) the degree of deformity correction at any single level is limited, (2) the time necessary to accomplish this task is magnified with each additional level, (3) the blood loss and risk to neural elements is magnified with each additional treated level, and (4) significant expense is incurred from the combination of cages and screws necessary to complete the task. These drawbacks are indeed exemplified by the authors' data. The correction of scoliosis from a mean of $19.3^{\circ}$ to $7.7^{\circ}$ and relative lack of effect on sagittal angulation are reflective of mild scoliosis. Furthermore, the mean operative time of $7.2 \mathrm{~h}$ is significant in the elderly population. The advantages of PLIF are the high fusion rates and excellent neural decompression, also seen in this series, and I am honestly surprised at the low reported complication rates given this magnitude of surgery. These factors have led our orthopedic colleagues to favor osteotomies as a method for more efficient and effective deformity correction.

PLIF and other interbody techniques may ultimately reemerge as an important method for the treatment of spinal deformities, but in the setting of minimally invasive spinal surgery. I think to this end, the authors have provided us with a valuable contribution to the literature by validating the PLIF technique as an acceptable and safe method for treating these patients.

\section{Conflicts of interest None.}

